\title{
PROSPECTS FOR AXION DETECTION
}

\author{
John Moody \\ Institute for Theoretical Physics, UCSB
}

Axions ${ }^{1}$ are a natural consequence of the only known solution of the "Strong CP Problem." 2 The axion represents a minimal $U(1)$ extension to the standard $S U(3) \times S U(2) \times U(1)$ model of the strong and electroweak interactions and furthermore appears very frequently in the contexts of SUSY GUTs, Kaluza-Klein, Superstring, Technicolor, and other unified models. As such, the axion is perhaps the best motivated conjectured particle.

Constraints from nuclear and particle physics experiments ${ }^{3}$ and stellar evolution $^{4}$ have suggested that the axion must have a very low mass and must interact extremely weakly with ordinary matter. Such weakly-interacting axions are known as "invisible axions"; 5 they are predicted to have masses of less than $10^{-2} \mathrm{eV}$. The fact that these axions are so weakly interacting means it is not possible to detect them using the traditional techniques of particle physics.

Invisible axions may play a central role in cosmology. If the mass of the axion is of order $\sim 10^{-5} \mathrm{eV}$, then standard axion cosmology scenarios predict that a primordial background of axions provides the closure density. ${ }^{6}$ This axion background is formed while the universe is at a temperature of $\sim 10^{12} \mathrm{GeV}$; since the axion background interacts very weakly with ordinary matter, it rapidly departs from thermal equilibrium as the universe expands. The axion background behaves as a high occupation number (classical), coherent, oscillating pseudoscalar field, and has been rightfully dubbed the "cosmic harmonic oscillator." Because axions departed from thermal equilibrium at a very early epoch, they provide a cold dark matter background for galaxy formation. If our galactic halo is composed of axions, the local number density is expected to be $\sim 10^{13}$ per $\mathrm{cm}^{3}$.

The hope that axions can be detected is based not upon single particle interactions but rather upon newly proposed macroscopic techniques in which large numbers of axions and conventional particles interact coherently. There are two classes of experiments: those which search for galactic halo axions and those which attempt to measure macroscopic forces mediated by virtual axions.

Galactic halo axions can be detected by converting them to microwave photons. Sikivie first suggested that this be accomplished in a microwave cavity via the axion-photon-photon coupling $(a \mathbf{E} \cdot \mathbf{B})$ by using a background magnetic field as a catalyst. ${ }^{7}$

In such an experiment, non-relativistic axions, say of mass $10^{-5} \mathrm{eV}$, are converted coherently into a microwave signal of frequency $2.4 \mathrm{GHz}$ which is observed classically. The difficulty in such experiments stems from our ignorance of the precise value of the axion mass, which requires that several orders of magnitude in frequency be searched. Several authors have analyzed in detail the sensitivity required for such search experiments. ${ }^{8,9}$ Given present technology, a halo density of axions may be detectable, but these experiments will not be sensitive enough to categorically rule-out axions as being important for cosmology. At this writing, 
three experimental groups are building magnetic field coupled detectors, ${ }^{10}$ and certain improvements in the original designs of Sikivie have been suggested. ${ }^{11}$

The inherent difficulty of these experiments suggests the need to examine other possibilities. It was recently proposed that larger conversion rates could be achieved in principle by using aligned electron spins as the catalyst. ${ }^{12,8}$ Experiments based upon electron spins are difficult to analyze, however, because the conversion rates depend sensitively upon material properties. ${ }^{13}$ Various possibilities have been suggested including the use of exotic ferrites, exotic paramagnetic salts, spin-polarized conductors, ferromagnetic resonance in YIG, and so on, but no workable schemes have emerged as yet. ${ }^{14}$

Experiments to detect axions via the macroscopic forces which they mediate $\mathrm{e}^{15}$ provide an independent test for the existence of those axions which happen to be of the greatest interest cosmologically, those with masses in the range around $10^{-5} \mathrm{eV}$. Such axions are expected to mediate forces over a range given by their Compton wavelength $\lambda \sim 2 \mathrm{~cm}$. Three types of forces are possible: a Yukawa force between nucleons, a tensor force between spins, and a very novel $P$ - and $T$ violating force between nucleons and spins. The presence of these forces can be probed by using the extremely sensitive techniques of experimental gravity.

\section{REFERENCES}

1. S. Weinberg, Phys. Rev. Lett. 40, 223 (1977); F. Wilczek, Phys. Rev. Lett. 40, 279 (1977).

2. R.D. Peccei and H. Quinn, Phys. Rev. Lett. 38, 1440 (1977).

3. T. Donnelly, et al. , Phys. Rev. D18, 1607 (1978).

4. M. Fukugita, S. Watamura, M. Yoshimura, Phys. Rev. Lett. 48, 1522 (1982), and Phys. Rev. D26, 1840 (1982); L. Krauss, J. Moody, F. Wilczek, Phys. Lett. 144B, 391 (1984); N. Iwamoto, Phys. Rev. Lett. 53, 1198 (1984); D. Morris (LBL report 18690,1984$)$.

5. J.E. Kim, Phys. Rev. Lett. 43, 103 (1979); M. Dine, W. Fischler, and M. Srednicki, Phys. Lett. 104B, 199 (1981); M. Shifman, A. Vainshtein, and V. Zakharov, Nucl. Phys. B166, 493 (1980).

6. J. Preskill, M.B. Wise, and F. Wilczek, Phys. Lett. 120B, 127 (1983); L.F. Abbott and P. Sikivie, Phys. Lett. 120B, 133 (1983); M. Dine and W. Fischler, Phys. Lett. 120B, 137 (1983).

7. P. Sikivie, Phys. Rev. Lett. 51, 1415 (1983), and 52, 695, (1984).

8. L. Krauss, J. Moody, F. Wilczek, and D. Morris, ITP preprint NSF-ITP-85-76.

9. P. Sikivie, University of Florida preprint (1985).

10. A. Melissinos, et al. , private communication (1985); P. Sikivie, N. Sullivan, D. Tanner, Experimental proposal (1984); P. Lubin, D. Morris and C. Pennypacker, Experimental Proposal (1985).

11. D. Morris, LBL Report LBL-17915 (1984).

12. L. Krauss, J. Moody, F. Wilczek, and D. Morris, ITP preprint NSF-ITP-85-08.

13. J. Slonczewski, IBM preprint (1985); private communications of C. Bennett, S. Coleman and N. Fortson.

14. Private communications of C. Bennett, G. Chapline, N. Fortson, J. Moody, D. Morris, J. Slonczewski, N. Snyderman, and F. Wilczek.

15. J. Moody and F. Wilczek, Phys. Rev. D30, 130 (1984). 